\title{
Phase-field simulations of nuclei and early stage solidification microstructures
}

\author{
B Nestler, M Selzer and D Danilov \\ Institute of Materials and Processes (IMP), Karlsruhe University of Applied Sciences, \\ Moltkestrasse 30, 76133 Karlsruhe, Germany
}

Received 22 April 2009, in final form 21 September 2009

Published 27 October 2009

Online at stacks.iop.org/JPhysCM/21/464107

\begin{abstract}
To investigate the local properties of heterogeneous nuclei on substrates, a phase-field model is extended to incorporate volume constraints and a third order line tension in the gradient free energy density formulation. The new model is applied to sessile drop simulations of $\mathrm{Cu}$ nuclei on Ni substrates to precisely analyse 3D equilibrium shapes and diffusion processes across the phase boundaries. In particular, the formalism with higher order potentials is used to investigate the length-scale dependent effect of the line tension on Young's force balance at triple lines in 3D. The employment of parallel and adaptive simulation techniques is essential for three-dimensional numerical computations. Early stage solidification microstructures of cubic Ni crystals are simulated by scale-bridging molecular dynamics (MD) and phase-field (PF) simulations. The domain of the PF computations is initialized by transferring MD data of the atomic positions and of the shape of the nuclei. The combined approach can be used to study the responses of microstructures upon nucleation.
\end{abstract}

(Some figures in this article are in colour only in the electronic version)

\section{Introduction}

The phase-field (PF) approach is a powerful methodology to describe phase transition phenomena (see recent reviews [1, 2]). This approach has been used to model solidification, microstructure formation in solids and motion of grain boundaries. PF models include formulations for the case of a pure substance [3], for multicomponent systems [4-6], for polycrystalline structure $[7,8]$ as well as for the phase transition where multiple different phases are involved: eutectic [9-11], peritectic [10] and monotectic [12] systems.

In PF models the individual phases are distinguished by one or more so-called phase fields. In different phase regions the phase fields attain different values, and interfaces are modelled by a diffuse interface. The phase fields, and also all other quantities, do not jump across the interface. They change continuously in a very thin transition layer (the diffuse interface), e.g. for a solid-liquid phase transition one can choose a phase field taking the value 1 in the solid and 0 in the liquid and across the interface the phase field varies from 1 to 0 .

According to the type of formulation, the following classes of PF models can be distinguished:

- models that involve a single scalar phase field [3],
- models that involve multiple or vector order parameters to treat multiphase transitions [13, 14],

- models derived from thermodynamic formulations [4, 14],

- models formulated for quasi-equilibrium processes [5, 15],

- models including a formulation for homogeneous and heterogeneous nucleation [16-20],

- models formulated for large derivations from local equilibrium [21, 22].

From a mathematical point of view, the PF models can be considered as a regularization of sharp interface problems. From a numerical point of view, the PF models provide an excellent method for computing complex geometrical patterns in moving boundary problems, even when the topology of the phase domains is changing during the evolution. As a phenomenological approach, the PF models cannot predict such interfacial properties as interfacial energy or kinetic coefficients and their anisotropies. These quantities are determined by effects and structures on atomic length and timescales which are averaged out in the PF description due to homogenization. In order to provide quantitative modelling, a combined approach [23] has been suggested. The properties of the solid-liquid interface are determined by molecular dynamics (MD) simulations and then transferred as input parameters into PF modelling. One of the modern directions in 
the development of PF models is the simulation of nucleation. The nucleation process takes place between atomistic and mesoscopic scales, because the size of the nucleus is atomistic with some tens or hundreds of atoms, but the nucleation time is mesoscopic.

In sections 2 and 3 of this paper we use a modified $\mathrm{PF}$ model to study diffusion processes and equilibrium force balance conditions of heterogeneous nuclei on substrates. Based on a combined scale-bridging MD and PF approach, we consider the evolution from nuclei to early stage solidification microstructures in section 4 . To compute the growth dynamics by PF simulations, the initial shape of the nuclei are taken from MD data of the atomic positions.

\section{Phase-field model to analyse heterogeneous nucleation}

\subsection{Formulation of volume constraints}

To investigate a liquid droplet on a solid substrate surrounded by a vacuum phase and effects arising at the contact boundary between the phases, we adopt a recently formulated PF model for multiple phases with volume constraints [24]. This model formulation allows us to maintain preserved volume fractions for some (or all) phases in the system. The method can be used to examine diffusion across the liquid-solid boundary as well as the influence of an additional triple line tension contribution to the energy balance and to the equilibrium contact angles. Without losing generality, we assume that the volume of the first $A$ of $N$ phase fields is conserved, whereas the parameters $\phi_{\alpha}, \alpha=A+1, \ldots, N$ should remain non-conserved. To ensure volume constraints, a new bulk free energy density

$$
g(\phi)=\sum_{\alpha=1}^{N} \chi_{\alpha} h\left(\phi_{\alpha}\right)
$$

is added to the functional acting as a force that counterbalances volume changes. A mixture between the additional contributions $\chi_{\alpha}$ of the different phases in the diffuse interface region is assumed by applying a polynomial interpolation function $h(\phi)$, e.g. $h(\phi)=\phi^{2}(3-2 \phi)$. As a result, the PF equations are modified by an additional force term $g, \phi_{\alpha}\left(\phi_{\alpha}\right)=$ $\chi_{\alpha} h, \phi_{\alpha}\left(\phi_{\alpha}\right)$ reading

$$
\tau \varepsilon \frac{\partial \phi_{\alpha}}{\partial t}=r h s_{\alpha}-\lambda-g, \phi_{\alpha}\left(\phi_{\alpha}\right)-\Lambda, \quad \alpha=1, \ldots, N,
$$

where $g, \phi_{\alpha}$ denotes the derivative of the function $g(\phi)$ with respect to $\phi_{\alpha}$. The contribution $g, \phi_{\alpha}$ requires an additional Lagrange multiplier $\Lambda$. The parameter $\varepsilon$ characterizes the thickness of the diffuse interface, $\tau$ is a kinetic coefficient that may, in general, be anisotropic, $r h s_{\alpha}$ contains the variational derivatives of the entropy density contributions and $\lambda$ is the Lagrange multiplier due to the constraint $\sum_{\alpha=1}^{N} \phi_{\alpha}=1$. Using the premise of a vanishing time derivative of the phase volume $\frac{\partial}{\partial t} V_{\alpha}=0=\int_{\Omega} \frac{\partial}{\partial t} \phi_{\alpha} \mathrm{d} x$ for the preserved phases $\alpha=1, \ldots, A$, the value of the correction force term can be determined. Differentiating between the case of preserving all of the phase volumes or only a number $A<N$ of them, we get

$$
\chi_{\alpha}= \begin{cases}\frac{1}{H_{\alpha}}\left(R_{\alpha}+\frac{1}{N-A} \sum_{\beta=1}^{A} R_{\beta}\right) & \text { for } 1 \leqslant A<N \\ \frac{R_{\alpha}}{H_{\alpha}} & \text { for } A=N .\end{cases}
$$

For all non-conserved PF parameters $\phi_{\alpha}(\alpha=A+$ $1, \ldots, N)$ the correction force must vanish: $\chi_{\alpha}=0$. In equation (3) the following definitions are used:

$$
\begin{gathered}
R_{\alpha}=\int_{\Omega}\left(r h s_{\alpha}-\lambda\right) \mathrm{d} x \quad \text { and } \\
H_{\alpha}=\int_{\Omega} h_{, \phi_{\alpha}}\left(\phi_{\alpha}\right) \mathrm{d} x .
\end{gathered}
$$

The nonlocal terms $R_{\alpha}$ and $H_{\alpha}$ include contributions of the complete domain $\Omega$. During the numerical simulations, they must be computed algorithmically after all right-hand sides of the PF equations for all grid points are known: first a timestep of equation (2) without correction is calculated for the complete domain $\Omega$, and afterwards the corrections $g, \phi_{\alpha}$ and $\Lambda$ due to the volume constraints are computed iteratively before the time update is completed.

\subsection{Formulation of a line tension energy density}

In analogy with an interface between two phases in the sharp interface limit, the coexistence line of three phases may have a contribution to the energy/entropy balance. From MD predictions [25], an additional energy of triple line order is essentially of relevance for small (nanometer) length scales. To account for this effect in the multiphase-field formulation [26], the anisotropic gradient energy density $a(\phi, \nabla \phi)$ in the functional is extended by a third order term $\sim \theta_{\alpha \beta \eta} \phi_{\eta}$ of the form

$$
a(\phi, \nabla \phi)=\sum_{\alpha<\beta} \gamma_{\alpha \beta}\left(1+\sum_{\eta \neq \alpha, \beta} \theta_{\alpha \beta \eta} \phi_{\eta}\right)\left[a_{\alpha \beta}\left(q_{\alpha \beta}\right)\right]^{2}\left|q_{\alpha \beta}\right|^{2},
$$

where the parameters $\theta_{\alpha \beta \eta}$ determine the energy contribution of the coexistence line between three phases $\alpha, \beta, \eta$. The additional energy only contributes in regions where all three phases are present, i.e. $\phi_{\alpha} \neq 0, \phi_{\beta} \neq 0$ and $\phi_{\eta} \neq 0$, converging in the sharp interface limit to the coexistence line in $3 \mathrm{D}$. In this formulation of the gradient energy density, $\gamma_{\alpha \beta}$ represents the surface energy density and $a_{\alpha \beta}\left(q_{\alpha \beta}\right)$ its anisotropy. By $q_{\alpha \beta}$, we denote generalized gradient vectors $q_{\alpha \beta}=\phi_{\alpha} \nabla \phi_{\beta}-\phi_{\beta} \nabla \phi_{\alpha}$.

\section{Simulations of sessile drop experiments}

\subsection{Modelling of the diffusion process}

The PF simulations are applied to sessile drop experiments where a $\mathrm{Cu}$ droplet $(\mathrm{L})$ is placed on top of a solid substrate (S), both surrounded by a vacuum (V). The droplet and the substrate form a free surface with the vacuum and the shape of this surface is varying in time. In order to 
a)

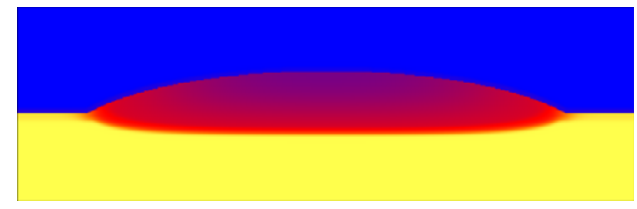

c)

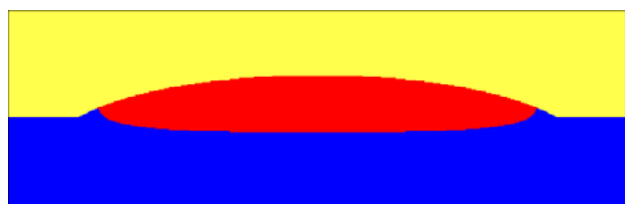

b)

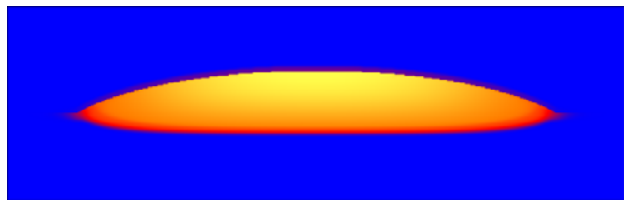

d)

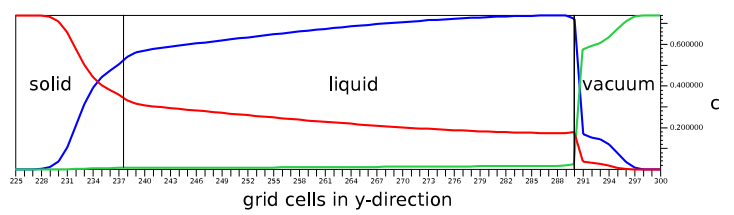

Figure 1. Concentration profiles of (a) $\mathrm{Ni}$ and (b) $\mathrm{Cu}$ across the sessile drop. (c) Illustration of the liquid droplet (red/light gray) on the solid substrate (blue/dark gray) surrounded by the vacuum (yellow/white). (d) Concentration profiles of the Ni (red/middle line), $\mathrm{Cu}$ (blue/top line) and $\mathrm{V}$ (green/bottom line) component in the $y$-direction.

Table 1. Melting temperatures $T_{i}^{\alpha}$ and latent heats $L_{i}^{\alpha}$.

\begin{tabular}{|c|c|c|c|c|c|c|}
\hline & $T_{\mathrm{Ni}}(\mathrm{K})$ & $T_{\mathrm{Cu}}(\mathrm{K})$ & $T_{\mathrm{V}}(\mathrm{K})$ & $L_{\mathrm{Ni}}\left(\mathrm{J} \mathrm{m}^{-3}\right)$ & $L_{\mathrm{Cu}}\left(\mathrm{J} \mathrm{m}^{-3}\right)$ & $L_{\mathrm{V}}\left(\mathrm{J} \mathrm{m}^{-3}\right)$ \\
\hline S & 1728 & 1358 & 1000 & $2350 \times 10^{6}$ & $1728 \times 10^{6}$ & $5728 \times 10^{6}$ \\
\hline $\mathrm{L}$ & 1728 & 1358 & 1000 & 0 & 0 & $5728 \times 10^{6}$ \\
\hline V & 1000 & 1000 & 1000 & $5728 \times 10^{6}$ & $5728 \times 10^{6}$ & $-5728 \times 10^{6}$ \\
\hline
\end{tabular}

eliminate numerical tracking of the surfaces with a vacuum, we represent the vacuum by an additional phase $\phi_{\mathrm{V}}$ in the $\mathrm{PF}$ model. Furthermore, we introduce a vacuum component $\mathrm{V}$ with concentration $c_{\mathrm{V}}$. The bulk free energy driving the diffusion process is described by an isothermal ideal-solution approximation

$$
f(T, c, \phi)=\sum_{i=1}^{3} \sum_{\alpha=1}^{3} c_{i} L_{i}^{\alpha} \frac{T-T_{i}^{\alpha}}{T_{i}^{\alpha}} h\left(\phi_{\alpha}\right)+\sum_{i=1}^{3} \frac{R T}{v_{m}} c_{i} \ln \left(c_{i}\right),
$$

where the summation index $i=1,2,3$ runs over the three concentration components and $\alpha=1,2,3$ numbers the three phases in the system. The melting temperatures $T_{\mathrm{Ni}, \mathrm{Cu}}^{\mathrm{S}, \mathrm{L}}$ and latent heats $L_{\mathrm{Ni}, \mathrm{Cu}}^{\mathrm{S}, \mathrm{L}}$ of $\mathrm{Ni}$ and $\mathrm{Cu}$ in the solid and liquid are physical parameters related to the phase diagram. The quantities $T_{\mathrm{V}}^{V}$ and $L_{\mathrm{V}}^{V}$ are set to model data related to the vacuum phase and the vacuum component. The values for $T_{\mathrm{V}}^{V}$ and $L_{\mathrm{V}}^{V}$ (see table 1 ) are chosen such that the resulting driving forces guarantee conservation of $\mathrm{Ni}$ and $\mathrm{Cu}$ atoms in the solid and liquid phases. The diffusion of $\mathrm{Ni}$ and $\mathrm{Cu}$ into the vacuum phase is suppressed, and simultaneously the vacuum component is restricted to the region of the vacuum phase.

The values of surface energy between L, S and V are taken from the experimental measurements given in [27, 28]. The simulations start with the liquid droplet of pure $\mathrm{Cu}$ lying on the solid substrate of pure $\mathrm{Ni}$ at a temperature $T_{\mathrm{Cu}}<T=$ $1540 \mathrm{~K}<T_{\mathrm{Ni}}$. At the boundary between solid and liquid, the diffusion leads to a change of the local alloy composition and the solid substrate melts slightly as can be seen in the snapshots of figure 1 . In the vicinity of the triple junctions, the solid phase grows from the Ni substrate toward the liquid droplet, strongly influenced by the contact angle condition between solid, liquid and vacuum. A similar lifting of the triple junctions is observed experimentally. Figure 1 also contains the concentration profiles of the three components at an advanced timestep of the diffusion process.

\subsection{Analysis of the effect of a line tension contribution}

In section 4 we will investigate the effect of the additional line tension contribution in equation (5) by 3D phase-field simulations. The initial configuration is a setup with three volume preserved phases $\mathrm{L}, \mathrm{S}$ and $\mathrm{V}$ with equal isotropic surface tensions corresponding to a $120^{\circ}$ angle condition at the triple junction. The first simulation ran under a zero line tension condition, i.e. $\theta_{\mathrm{SLV}}=0$, and until the equilibrium shape of the droplet was established (figure 2 (brown line) and figure 3(a)). The final structure of the equilibrated droplet was used to initialize the computational domain for the simulations with non-zero line tensions $\theta_{\mathrm{SLV}}=10,20$ and 50. The final $3 \mathrm{D}$ shape of the droplet with $\theta_{\mathrm{SLV}}=50$ is shown in figure $3(\mathrm{~b})$. By evaluating two-dimensional cuts in vertical and horizontal planes (figures 2(a) and (b)), the influence of the line tension on the curvature and morphology of the phase boundaries can be seen. As a result of the additional contribution to the curvature, the diameter of the triple line is reduced. In forthcoming work, the proposed predictions of the phase-field simulations concerning the effect of the line tension will be evaluated by MD simulations and by precise sessile drop experiments. It is planned to concisely analyse the dependence of the line tension on the size of the droplet.

\section{Scale-bridging MD and PF simulations from atomic nuclei to microstructures}

As a phenomenological approach, PF models cannot predict interfacial properties such as interfacial energy, kinetic coefficients and anisotropies. These quantities are determined by effects and structures on atomic length and timescales which are averaged out in the phase-field description due to homogenization. In order to provide quantitative modelling, a combined approach of MD and PF simulations is applied to nucleation and growth of a pure $\mathrm{Ni}$ crystal. This is a combination of the two techniques operating on different scales in a transfer of early stage solidifying microstructures from $\mathrm{MD}$ to PF in a calibration of a PF parameter (such as the interfacial thickness) and in a determination of thermophysical data for PF simulations by MD. The methodology for bridging the scales involves large scale MD simulations and small scale PF simulations employed at the same lengthscales and 

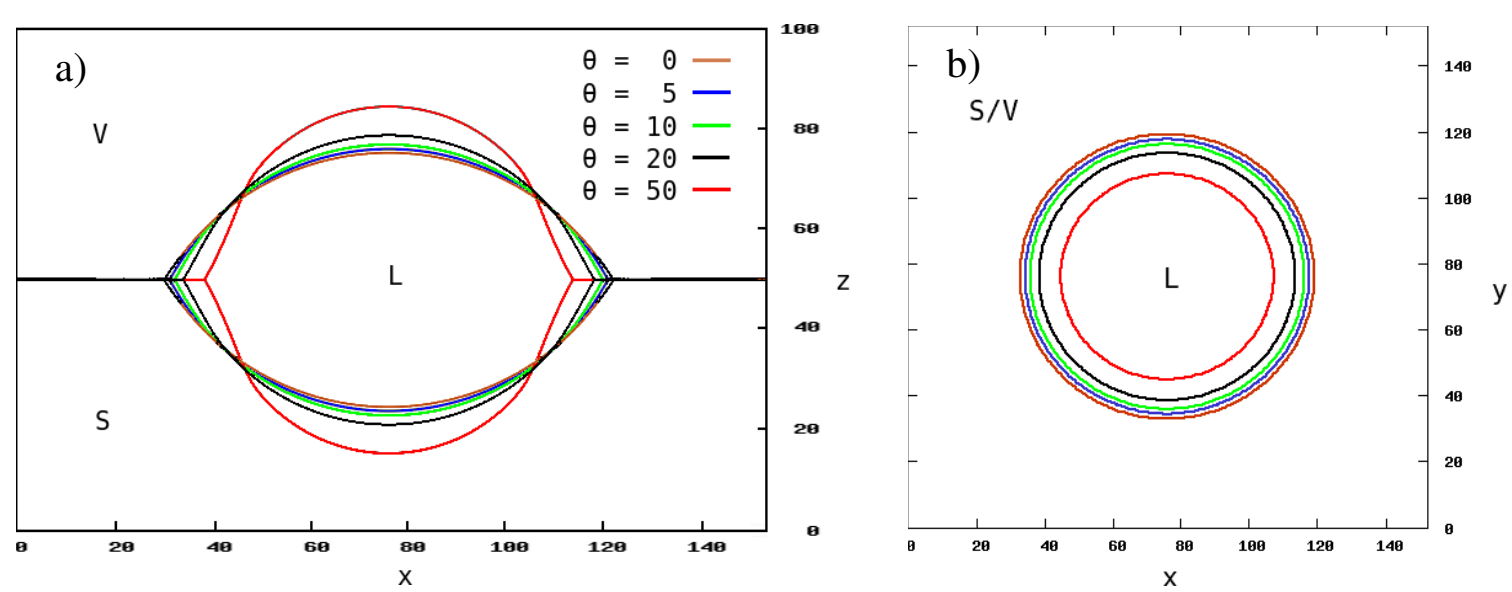

Figure 2. Diagrams with isolines for $\theta_{\mathrm{SLV}}=0,10,20$ and 50 showing a cut (a) through the droplet and the surface and (b) a top view of the corresponding triple lines.
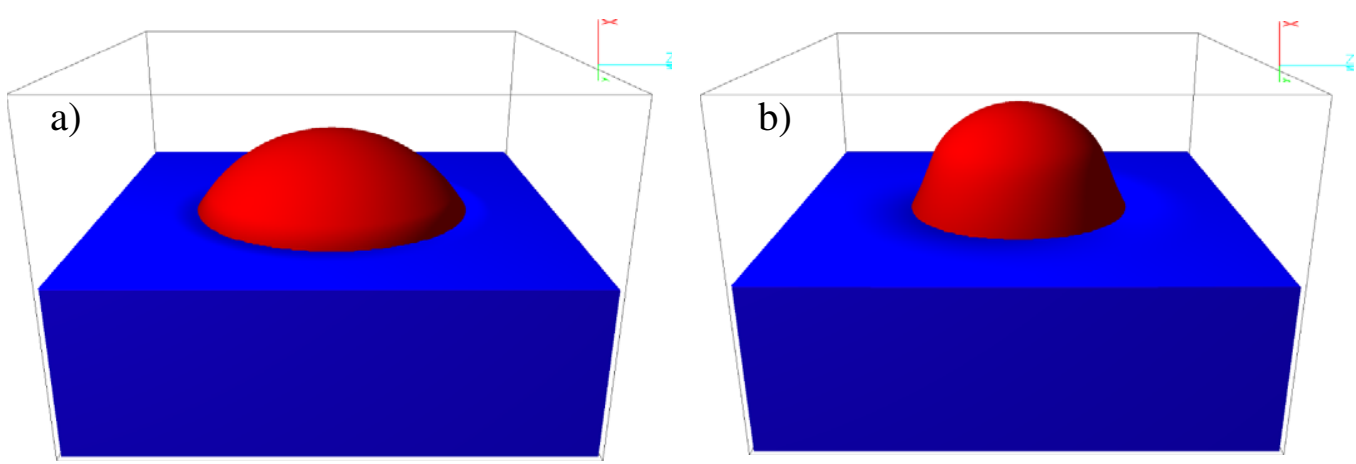

Figure 3. Effect of the new energy density contribution accounting for a tension of the triple line in 3D structures: (a) droplet on a substrate surface for $\theta_{\mathrm{SLV}}=0$ (no line tension) and (b) for $\theta_{\mathrm{SLV}}=50$.

timescales. The results of both methods are compared and benchmarked. For the MD simulations, the crystal-melt interface in the pure $\mathrm{Ni}$ system is modelled by the interatomic potential using the embedded atom method and the system is considered as an isothermal, isobaric NPT ensemble with 32000 atoms, [29, 30]. The PF model is based on a standard formulation presented in $[13,26]$, for example.

The MD simulations were set up in a domain with an initial Ni melt undercooled by $300 \mathrm{~K}$. A nucleus forms naturally by homogeneous nucleation and grows to extend approximately $50 \AA$ in each dimension.

In temporal continuation of this early stage solidification, the PF method takes over and uses information about the interfacial structure and atomic positions provided by the final timestep of the MD simulation.

To model the solid-liquid interface and the growth of the initial nucleus on atomistic scales, we calculate the positions of the atoms by a local order parameter $Q_{6}(x)$ in the standard form introduced by Steinhardt et al [31]. A detailed description of the mathematical expression is given in section 3.1 of a recent publication [32]. The order parameter $Q_{6}(x)$ distinguishes crystalline and liquid type particles and hence defines the crystal-melt interface. The profile of the $Q_{6}(x)$ order parameter shows a smooth transition from the crystal to the liquid phase state extending over an atomic distance of about $6 \AA$ at thermodynamic equilibrium. The discrete data points of the $Q_{6}$ order parameter and the atomistic interface thickness are used to fit the tanh-profile of the diffuse interface of the phase-field model.

For that, data from large scale MD simulations provided by [25] describing the positions of atoms are converted into the formulation and format required for PF simulations. The PF simulations use the transferred data to continue the computation of microstructure formation.

In accordance with the initial MD condition, the PF model is adopted in isothermal approximation for pure substances, i.e. the set of order parameters reduces to one phase field $\phi=\phi_{S}$. By overlapping the simulation windows, the methodology covers the evolution from nucleation to dendrite microstructures.

The $Q_{6}$ order parameter is rescaled to match the transition of the PF variable $\phi$ from 0 in the melt to 1 in the crystal and converted into input data for the PF model. From an ansatz function $\Psi(x)=A+\frac{B}{2}\left(1-\tanh \frac{3}{2} \frac{x-x_{0}}{\epsilon}\right)$, the fitting of the MD data points gives the values $\epsilon=3 \AA$ for the PF model parameter $\epsilon$ that determines the width of the diffuse interface, so the variation of the $\mathrm{PF}$ is from $\phi=1$ to 0 over $2 \epsilon=$ $6 \AA$. Using this value, the PF reproduces the same interfacial thickness as in the MD simulations. The interfacial properties are determined at the final timestep of the MD computation and are used as a constant input parameter for the subsequent $\mathrm{PF}$ model. The complete domain for the PF computations is 

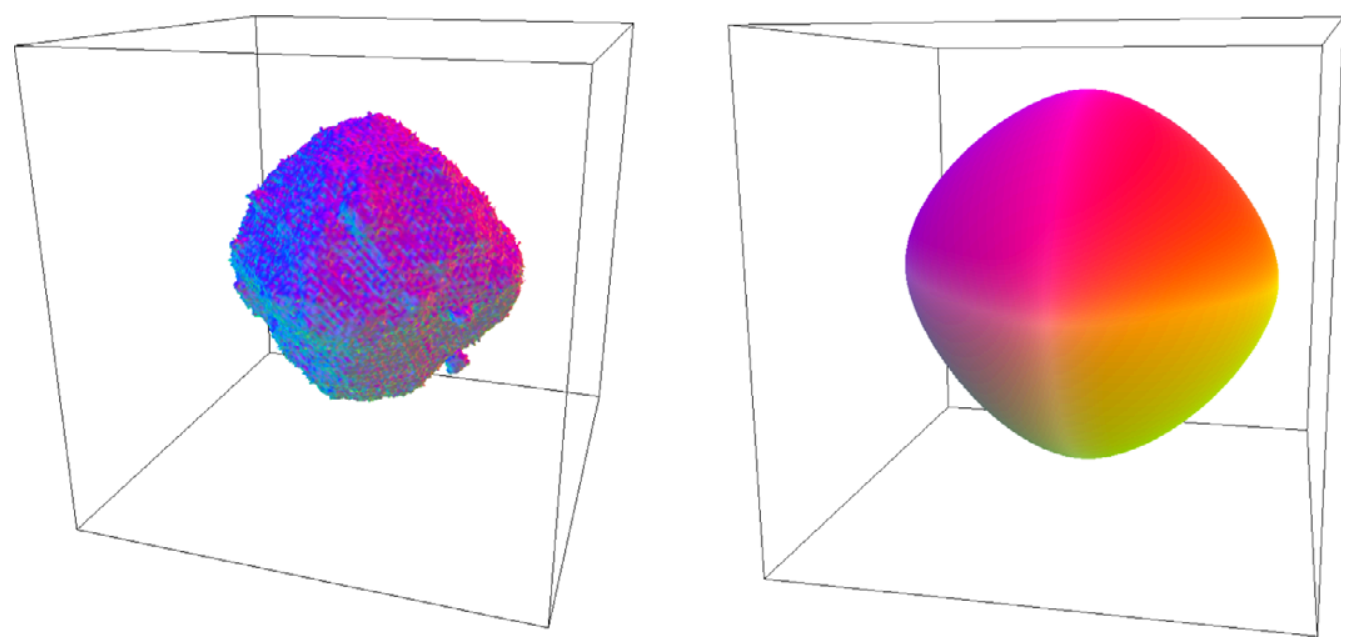

Figure 4. Initial nucleus of the Ni crystal transferred from MD simulation (left) and corresponding PF simulation at an early stage of solidification (right). The spatial extension of the computational box refers to $70 \times 70 \times 70 \AA^{3}$.

filled by transferring the positions of the bulk regions from the final timestep of the MD simulation and by interpolating the solid-liquid interface with a tanh-profile and interfacial width mechanism according to the filling description above. The scale-bridging maps the spatial coordinates of the final MD microstructure onto the PF data and shows the successive time evolution from nucleation on small atomic scales to grown nuclei on large atomic scales up to dendritic microstructure on mesoscopic scales.

After the filling, the PF equations are solved in the usual manner for the evolution in the PF domain. Further physical parameters such as the latent heat $L=0.0168 \mathrm{eV}^{-3}$ (DLR), melting point $T_{\mathrm{m}}=1748 \mathrm{~K}$ (DLR) and the kinetic coefficient $\tau^{0}=0.3692 \mathrm{~m} \mathrm{~s}^{-1} \mathrm{~K}^{-1}$ are directly taken as input parameters from the corresponding MD simulations. The value for the interfacial energy and the anisotropy coefficients $\epsilon_{c}=0.027$ and $\epsilon_{k}=0.169$ are adopted from MD simulations in [33, 34]. The isothermal undercooling condition of $300 \mathrm{~K}$ refers to the constant system temperature of $T=1428 \mathrm{~K}$ assumed in both MD and PF simulations. In figure 4 it can be seen that, due to the general principle of minimization of energy and, respectively, curvature in the diffuse interface formulation of the PF model, the rough shape of the MD nucleus smoothens and the growing crystal in the PF simulation forms a convex shape with cubic crystal symmetry at this early stage of solidification. In contrast, the MD simulations exhibit different shapes of atom positions and nuclei, whereas the PF simulations average out the rough structures resulting in almost the same shape of the crystal, independently of the randomly distributed initial positions of atoms. Under non-isothermal conditions, the crystal further develops side branches and evolves in a dendritic morphology.

\section{Conclusions}

A new formulation of a line tension energy density is incorporated in a general PF model for multiphase systems including an extension for phases with preserved volume.
In applications of the PF model to sessile drop settings of $\mathrm{Ni}$ and $\mathrm{Ni}-\mathrm{Cu}$, a ternary system has been constructed to model the vacuum phase surrounding the droplet. The diffusion profiles of $\mathrm{Ni}$ and $\mathrm{Cu}$ in the phases is discussed. Using the new energy formulation, the PF simulations allow predictions of the line tension on the curvature of the triple line and on the angle condition of the equilibrium force balance. In future research, the diffusion profiles as well as the predictions of the line tension effect will be validated by MD simulations and sessile drop experiments. Furthermore, we developed a programming interface to conduct combined MD and PF simulations. The framework can be used to compute the processes of evolution from atomic nuclei to dendritic microstructures. The methodology also allows us to study the effect of nucleation on the solidification morphologies.

\section{References}

[1] Chen L-Q 2002 Phase-field models for microstructure evolution Annu. Rev. Mater. Res. 32 113-40

[2] Boettinger W J, Warren J A, Beckermann C and Karma A 2002 Phase-field simulation of solidification Annu. Rev. Mater. Res. 32 163-94

[3] Karma A and Rappel W-J 1998 Quantitative phase-field modeling of dendritic growth in two and three dimensions Phys. Rev. E 57 4323-49

[4] Bi Z and Sekerka R F 1998 Phase-field model of solidification of a binary alloy Physica A 261 95-106

[5] Echebarria B, Folch R, Karma A and Plapp M 2004 Quantitative phase-field model of alloy solidification Phys. Rev. E 70061604

[6] Nestler B, Garcke H and Stinner B 2005 Multicomponent alloy solidification: phase-field modeling and simulations Phys. Rev. E 71041609

[7] Gránásy L, Pusztai T and Warren J 2004 Modelling polycrystalline solidification using a phase field theory J. Phys.: Condens. Matter 16 R1205-35

[8] Posztai T, Bortel G and Gránásy L 2005 Phase field modeling of polycrystalline freezing Mater. Sci. Eng. A 413/414 412-7

[9] Karma A 1994 Phase-field model of eutectic growth Phys. Rev. E 49 2245-50 
[10] Nestler B and Wheeler A A 2000 A multi-phase-field model of eutectic and peritectic alloys: numerical simulation of growth structures Physica D 138 114-33

[11] Folch R and Plapp M 2005 Quantitative phase-field modeling of two-phase growth Phys. Rev. E 72011602

[12] Nestler B, Wheeler A A and Ratke L 2000 Phase-field model for solidification of a monotectic alloy with convection Physica D 141 133-54

[13] Steinbach I, Pezzolla F, Nestler B, Seesselberg M, Prieler R and Schmitz G J 1996 A phase field concept for multiphase systems Physica D 94 135-47

[14] Garcke H, Nestler B and Stinner B 2004 A diffuse interface model for alloys with multiple components and phases SIAM J. Appl. Math. 64 775-99

[15] Karma A 2001 Phase-field formulation for quantitative modeling of alloy solidification Phys. Rev. Lett. 8711570

[16] Gránásy L, Pusztai T and Hartmann E 1996 J. Cryst. Growth 167756

[17] Gránásy L and Pusztai T 2002 Diffuse interface analysis of crystal nucleation in hard-sphere liquid J. Chem. Phys. 11710121

[18] Gránásy L, Pusztai T, Tóth G and Jurek Z 2003 Phase field theory of crystal nucleation in hard sphere liquid J. Chem. Phys. 11910376

[19] Gránásy L, Börzsönyi T and Pusztai T 2002 Crystal nucleation and growth in binary phase-field theory $J$. Cryst. Growth 137-139 1813-7

[20] Gránásy L, Börzsönyi T and Pusztai T 2002 Nucleation and bulk crystallization in binary phase-field theory Phys. Rev. Lett. 88206105

[21] Wheeler A A, Boettinger W J and McFadden G B 1993 Phase-field model of solute trapping during solidification Phys. Rev. E 47 1893-909

[22] Ahmad N A, Wheeler A A, Boettinger W J and McFadden G B 1998 Solute trapping and solute drag in a phase-field model of rapid solidification Phys. Rev. E $583436-50$
[23] Bragard J, Karma A, Lee Y H and Plapp M 2002 Linking phase-field and atomistic simulations to model dendritic solidification in highly undercooled melts Interface Sci. 10 121-36

[24] Nestler B, Wendler F, Selzer M, Stinner B and Garcke H 2008 Phase-field model for multiphase systems with preserved volume fractions Phys. Rev. E 78011604

[25] Horbach J private communications

[26] Nestler B, Garcke H and Stinner B 2005 Multicomponent alloy solidification: phase-field modelling and simulations Phys. Rev. E 71041609

[27] Brillo J and Egry I 2004 Density and excess volume of liquid copper, nickel, iron, and their binary alloys Int. J. Mater. Res. 8 691-7

[28] Brillo J and Egry I 2005 Surface tension of nickel, copper, iron and their binary alloys J. Mater. Sci. $402213-6$

[29] Daw M S and Baskes M I 1983 Semiempirical, quantum mechanical calculation of hydrogen embrittlement in metals Phys. Rev. Lett. 501285

Daw M S and Baskes M I 1984 Embedded-atom method: derivation and application to impurities and other defects in metals Phys. Rev. B 296443

[30] Daw M S, Foiles S M and Baskes M I 1993 The embedded atom method: a review of theory and applications Mater. Sci. Rep. 9251

[31] Steinhardt P J, Nelson D R and Ronchetti M 1983 Bond-orientational order in liquids and glasses Phys. Rev. B 28784

[32] Nestler B, Danilov D, Guerdane H and Teichler H 2009 Bridging the gap between molecular dynamics simulations and phase-field modelling: dynamics of a

$\left[\mathrm{Ni}_{x} \mathrm{Zr}_{1-x}\right]_{\text {liquid }} \mathrm{Zr}_{\text {crystal }}$ solidification front J. Phys. D: Appl. Phys. 42015310

[33] Hoyt J J, Sadigh B, Asta M and Foiles S M 1999 Kinetic phase-field parameters for the $\mathrm{Cu}-\mathrm{Ni}$ system derived from atomistic computations Acta Mater. 47 3181-7

[34] Asta M, Hoyt J J and Karma A 2002 Calculation of alloy solid-liquid interfacial free energies from atomic-scale simulations Phys. Rev. B 66100101 